\title{
Estrategias y técnicas didácticas en entornos virtuales: análisis e importancia para docentes y estudiantes
}

\section{Teaching strategies and techniques in virtual environments:} analysis and importance for teachers and students Estratégias e técnicas de ensino em ambientes virtuais: análise e importância para professores e alunos

Marylin Tais Condori Polloyqueri

marylin.condori@upeu.edu.pe

https://orcid.org/0000-0003-0102-5456

Universidad Peruana Unión

Maribel Susan Alvarez Luque

maribel.alvarez@upeu.edu.pe

https://orcid.org/0000-0001-9652-2361

Universidad Peruana Unión
Yaneth Copatarqui Calisaya

yaneth.copatarqui@upeu.edu.pe

iD https://orcid.org/0000-0003-1641-9423

Universidad Peruana Unión

Jhonatan Chambi Condori

jhchambic@upeu.edu.pe

https://orcid.org/0000-0003-3711-6608

Universidad Peruana Unión

Neri Yesenia Rojas Marchan

ynrm01@gmail.com

iD https://orcid.org/0000-0002-0390-342x

Universidad Nacional Daniel Alcides Carrión, Perú

Recibido: 03 de enero de 2021

Aceptado: 06 de junio de 2021

\section{Resumen}

El presente suscribe el objetivo: subrayar la importancia de las estrategias y técnicas didácticas, en los entornos virtuales, para los docentes y los estudiantes, durante el proceso de enseñanza aprendizaje. La educación, a través de toda la historia, exige el uso de estrategias y técnicas didácticas, pertinentes y en respuesta a la problemática: educativa, académica y pedagógica; sin embargo, esta problemática del proceso enseñanza aprendizaje no ha sido solucionada. En conclusión, el uso de estas estrategias 
y técnicas es imprescindible, porque permite que los docentes mejoren sus estilos y habilidades de enseñanza, dejando, en consecuencia, a los estudiantes coronados de beneficios importantes, potenciando sus habilidades y el aprendizaje autónomo, en un espacio de mayor vinculación del docente y estudiante.

Palabras clave: Estrategias didácticas, técnicas didácticas, entornos virtuales.

\begin{abstract}
The present endorses the objective: to underline the importance of teaching strategies and techniques, in virtual environments, for teachers and students, during the teachinglearning process. Education, throughout history, requires the use of didactic strategies and techniques, pertinent and in response to the problem: educational, academic and pedagogical; However, this problem of the teaching-learning process has not been solved. In conclusion, the use of these strategies and techniques is essential, because it allows teachers to improve their teaching styles and skills, consequently leaving students crowned with important benefits, enhancing their skills and autonomous learning, in a space of greater bonding of the teacher and student.
\end{abstract}

Keywords: Didactic strategies, didactic techniques, virtual environments.

\title{
Resumo
}

O presente endossa o objetivo: sublinhar a importância das estratégias e técnicas de ensino, em ambientes virtuais, para professores e alunos, durante o processo de ensinoaprendizagem. A educação, ao longo da história, requer o uso de estratégias e técnicas didáticas, pertinentes e em resposta à problemática: educacional, acadêmica e pedagógica; Porém, esse problema do processo ensino-aprendizagem não foi resolvido. Conclui-se que a utilização dessas estratégias e técnicas é imprescindível, pois permite aos docentes aprimorar seus estilos e habilidades de ensino, consequentemente deixando os alunos coroados de importantes benefícios, valorizando suas competências e aprendizagem autônoma, em um espaço de maior vínculo do professor e estudante.

Palavras-chave: Estratégias didáticas, técnicas didáticas, ambientes virtuais.

\section{Introducción}

El encuentro frecuente de los protagonistas de la educación, en entornos virtuales, se ha constituido en parte de la vida; es decir, el docente y el estudiante, dejando al margen las labores académicas en su modalidad acostumbrada: la modalidad presencial. Los 
entornos virtuales determinan la condición imprescindible para el desarrollo educativo y formación profesional del estudiante. La incorporación de esto entorno en el sistema educativo ha generado cambios en el proceso enseñanza aprendizaje, incorporando metodologías, estrategias y técnicas didácticas.

Las instituciones educativas se han visto obligadas a la adaptación a estos entornos virtuales; han experimentado el acondicionamiento y desarrollo de nuevos modelos de enseñanza, cuyo uso de estrategias y técnicas didácticas han sido adaptadas a la situación actual. En la situación educativa, en la actualidad, los docentes se han visto en la necesidad de implementar y desarrollar habilidades, que les permitan el desenvolvimiento académico en estos entornos virtuales (Olivares et al., 2016).

Para Abarca (2015), “el surgimiento de nuevas tecnologías y formas de trabajo ha creado la necesidad de que los profesionales, y entre ellos muy especialmente los docentes, desarrollen habilidades y competencias para que puedan utilizar las herramientas tecnológicas de forma efectiva" (p.336). Por su parte, Palma et al., (2021) refieren: La incorporación de estrategias tecnológicas de forma virtual en el campo educativo, ha otorgado que el docente forme, renueve y genere nuevas destrezas, métodos, recursos y técnicas pedagógicas que ayuden a aumentar el nivel de calidad de los estudiantes por aprender y adquirir conocimientos (p.2).

La problemática de estos entornos virtuales revela que muchos docentes no han logrado plenamente la adaptación y desarrollo de habilidades tecnológicas; pues no se hace uso correcto de estrategias y técnicas didácticas, ya que difieren de las que se aplican en los espacios presenciales. Silva \& Maturana (2017) manifiestan que las técnicas didácticas tradicionales no desarrollan las habilidades en los estudiantes, por la diversidad de formas de aprendizaje y por la falta de acompañamiento, durante el seguimiento del aprendizaje.

El estudio tiene un espacio de actuación determinado por el objetivo: subrayar la importancia académica de las estrategias y técnicas didácticas, para los protagonistas principales y sustantivos del proceso enseñanza aprendizaje en un entorno virtual, donde se observen las habilidades, las capacidades, la autonomía y la creatividad. Para lograr este objetivo son desarrollados varios aspectos temáticos, con los cuales quedarán clarificadas las rutas educativas y académicas. 


\section{Estrategias didácticas}

\section{Conceptos}

Las estrategias didácticas involucran también procesos afectivos, cognitivos y procedimentales, generando, en consecuencia, la construcción y desarrollo del aprendizaje en los estudiantes; además, permiten que el docente asimile el proceso de enseñanza. Feo (2010) menciona: Las estrategias didácticas se definen como los procedimientos (métodos, técnicas, actividades) por los cuales el docente y los estudiantes, organizan las acciones de manera consciente para construir y lograr metas previstas e imprevistas en el proceso enseñanza y aprendizaje, adaptándose a las necesidades de los participantes de manera significativa (p.222).

Por lo tanto, las estrategias didácticas son métodos con propósitos e intenciones bastante definidos, poseen una diversidad de concepciones subjetivas, las cuales dependen de los recursos existentes y del contexto, donde se realiza el proceso. No solo una correcta exposición oral, en una clase, es suficiente, para sostener que una clase es correcta; son necesarias las estrategias didácticas, para la enseñanza-aprendizaje correcta y acorde. Las estrategias didácticas conforman un conjunto de herramientas, útiles para desarrollar el pensamiento crítico y creativo de los estudiantes, mientras aprenden los contenidos y temas de cada asignatura (Rosales, n.d.).

Delgado \& Solano (2009) refieren que los factores que influyen en la acción didáctica son: el docente o profesor, el discente o alumnado, el contenido o materia, el contexto del aprendizaje, las estrategias metodologías o didácticas.

\section{Tipos de estrategias didácticas}

Dentro del estudio de las estrategias didácticas, se abordan las estrategias de enseñanza y de aprendizaje.

\section{Estrategias de aprendizaje}

Estas estrategias forman parte de un grupo de procesos, cuyos procesos afectan el proceso de aprendizaje. En la actualidad, existen un sinnúmero de estrategias, adaptadas a los intereses y las necesidades de los estudiantes; estas estrategias facilitan y sintonizan la planificación, la organización y el control del proceso correspondiente. El uso de las estrategias de aprendizaje se realiza para lograr el objetivo del proceso enseñanza 
aprendizaje (Maria, 2020). "Estas estrategias de aprendizaje son las que designan las técnicas que se emplean en la resolución de tareas específicas a la hora de aprender" (Hoffman et al., 2017, p.540).

Estrategias de enseñanza

Pamplona et al. (2019) las definen: "metodología del docente para lograr que los contenidos, temáticas e información logren ser aprendidas por el estudiante y se genere el desarrollo de competencias" (p.14). Es decir, las estrategias de enseñanza son un conjunto de decisiones que toma un docente, para orientar la enseñanza, con el propósito de promover el aprendizaje de sus alumnos (Marsiglia et al., 2020).

\section{Técnicas didácticas}

\section{Conceptos}

Las técnicas didácticas son un grupo de procedimientos, los cuales aportan y contribuyen para el logro de un aprendizaje, cuyo uso facilita el proceso de formación de los educandos. Las técnicas didácticas pueden ser definidas: "estrategias que utiliza el docente para convertir el proceso de enseñanza en actividades que fomenten la participación activa del estudiante y lleven al aprendizaje" (Labrador \& Andreu, 2008, p.6).

Estas técnicas se solidifican para lograr un cambio en la forma de aprender; se centran básicamente sobre las actividades que deben realizar y el contenido teórico que deben aprender; esto faculta y permite realizar cambios durante el proceder de los docentes y de los estudiantes (Silva \& Maturana, 2017).

La técnica didáctica es un procedimiento didáctico que ayuda a realizar una parte del aprendizaje que se persigue con la estrategia. Mientras la estrategia abarca aspectos más generales del curso o de un proceso de formación completo, la técnica se enfoca a la orientación del aprendizaje en áreas delimitadas de la asignatura o curso. Dicho de otra manera, la técnica didáctica es el recurso particular, del cual se vale el docente para lograr los propósitos planeados desde la estrategia.

\section{Tipos de técnicas didácticas}

Delgado \& Solano (2009) especifican los siguientes tipos de estrategias didácticas, en el ámbito de la enseñanza virtual; a su vez ciertamente incluyen diversas técnicas para el desarrollo del proceso de enseñanza. Dichas técnicas son:

Técnicas centradas en la individualización de la enseñanza 
Se engloba encuentran, dentro de este marco, las técnicas adaptadas a las necesidades y los intereses de los estudiantes; por ejemplo, la recuperación de información, el trabajo individual con el uso de materiales interactivos, los contratos de aprendizaje, las prácticas, el aprendiz, las técnicas centradas en el pensamiento crítico y en la creatividad.

Precisamente para el uso de estas técnicas, el docente establece una relación directa con el estudiante, generando el progreso de este último. Se comparte las siguientes:

Recuperación de información. El estudiante construye su propio conocimiento, mediante la búsqueda y localización de información en Internet, ejerciendo el análisis y la valoración de la misma. El docente guía, da pautas, para la buena búsqueda de información; infunde en el estudiante la búsqueda de una información acertada, generando comparaciones entre las fuentes de búsqueda.

Trabajo con materiales multimedia interactivos. Se realiza para fomentar la creatividad, el pensamiento analítico y crítico, en los estudiantes. El docente solicita la realización de trabajos: ejercicios, casos prácticos y tutoriales; los revisa de acuerdo con los criterios regulados.

Contratos de aprendizaje. De acuerdo con esta técnica se establece un currículo adaptado a las necesidades educativas individuales; el estudiante construye sus conocimientos, mediante el abordaje de los temas, las herramientas, los procedimientos, los cuales incentivan el aprendizaje; los docentes establecen los objetivos concretos, para que el estudiante se esfuerce y logre superarse.

Prácticas. Se priorizan las prácticas de los estudiantes, a través de la red, controladas en forma constante por los docentes, para lograr la evolución de los primeros.

El aprendiz (apprenticeship). El estudiante interactúa con el experto. El docente está suficientemente capacitado, logrando transmitir y guiar al estudiante, quien ejerce dos roles dentro de un salón de clases: el mentorage, el estudiante guía a sus compañeros más inexpertos, es denominado el aprendiz colaborador, quien además orienta, guiar y realiza los trabajos individuales, en compañía, juntamente con sus compañeros.

Técnicas centradas en el pensamiento crítico. Se realizan actividades: selección y evaluación de la información o las soluciones potenciales, la organización de la misma. Por ejemplo, la creación de gráficos, los ensayos sobre pros y contras, los aspectos positivos y negativos, las síntesis de lluvia de ideas, los sumarios, las reflexiones, los esquemas, entre otros, con sentido crítico. 
Técnicas centradas en la creatividad. El docente actúa de gestor, para la distribución de actividades; realiza seguimiento a los procedimientos de solución de los estudiantes. Se desarrolla, en los alumnos, la habilidad creativa, con el propósito de abordar temas casuísticos o problemáticos, mediante imaginación, intuición, curiosidad, conexión con las experiencias previas, habilidad artística, etc.

\section{Técnicas para la enseñanza en grupo}

Son técnicas de un conocimiento en conjunto, grupal. Se precisa dos elementos con sus respectivas funciones: el primero, es el docente o estudiante, quienes hacen de expositor y el segundo, el grupo que recibe la información.

El grupo de atención a la clase impartida cumple determinadas responsabilidades; primero, en forma individual, para compartir sus ideas en forma grupal. Estas técnicas tienen el objetivo de juzgar críticamente los aportes de sus compañeros y obtendrán un trabajo más consolidado.

Estas técnicas son: exposición didáctica, preguntas al grupo, simposio, mesa redonda o panel, entrevista o consulta pública, tutoría pública, tablón de anuncios y exposiciones.

Exposición didáctica. Son sesiones de corta duración, actividades para reforzar lo aprendido. Exponen cierta diferenciación entre aspectos básicos y secundarios, en forma didáctica, mediante elementos de apoyo y demostrativos: elementos gráficos, visuales o auditivos, con pensamiento crítico de los estudiantes.

Preguntas al grupo. Primero, se delega investigar cierto tema a un grupo determinado, luego se le hace las preguntas. Esta técnica es bastante conocida, se practica en foros al inicio de las sesiones de clases, buscan el diálogo, se materializa en debates. Estimula participación, autovaloración; el docente detecta fortalezas, debilidades, refuerzan el aprendizaje.

Simposio. Los integrantes del grupo se preparan sobre diferentes puntos o aspectos de un mismo tema; según esta técnica grupal, los participantes exponen sus ideas; los estudiantes realizan preguntas o reflexiones. La mesa redonda es semejante al simposio; sin embargo, es mucho más formal; los expertos exponen sus conocimientos; también realizan una discusión. En el ámbito del panel, técnica conocida del foro, los integrantes realizan un diálogo en frente del grupo. 
Entrevista o consulta pública. Permite enriquecer los conocimientos; el primer paso es investigar en forma previa y por propia cuenta; el segundo, preparar las preguntas y reflexiones; el tercero, consultar al experto sobre el tema concreto.

Tutoría pública. El profesor dictará un curso, mediante la vía virtual, con el propósito de responder las interrogantes de los estudiantes.

Tablón de anuncios. Es un espacio, para que los estudiantes intercambien información, respuestas, problemas, experiencias, reflexiones y otros.

Exposiciones. Son asignadas por el docente, para que los estudiantes realicen investigaciones sobre determinados temas, generando análisis y síntesis; promueven la creatividad para las exposiciones, espacio para la discusión y profundización.

Técnicas centradas en el trabajo colaborativo.

En realidad priman imprescindiblemente las técnicas de colaboración; se promueve el trabajo en equipo, generando estructuras de comunicación. El trabajo es evaluado en forma constante, cuya estructura es establecida por el docente. Las técnicas más recomendadas son:

Trabajo en parejas. Se realiza entre dos miembros de un grupo, para realizar una entrevista, comparar resultados, intercambiar los trabajos, con el propósito de realizar la respectiva revisión, entre otros.

Lluvia de ideas. Tiene el fin: dar a conocer todas las ideas y conocimientos de cada participante; el docente es moderador, logrando la síntesis y conclusión. Las ideas son registradas en una hoja, con el propósito de no olvidar; luego se jerarquiza y clasifica las más idóneas; finalmente se obtiene la síntesis y conclusión.

Rueda de ideas. Se divide el grupo en otros pequeños subgrupos; cada integrante comunica sus ideas; solamente se selecciona 5, las más resaltantes.

Votación. La idea es someter a votación las ideas generadas, los diversos puntos de vista, todas las sugerencias de cada integrante, con la orientación del profesor.

Debate y foro. Se observa dos roles en interacción: el docente, moderador, da inicio, comunicando los mecanismos de la participación, evitando las dudas y hace el cierre del debate luego de la presentación de las conclusiones. El otro rol es los miembros del grupo, quienes comunican sus ideas, coincidentes u opuestas, con el propósito de contrastar los diversos puntos de vista. El debate y el foro constituyen técnicas formales. 
Controversia estructurada. Se trata de dividir al grupo en dos subgrupos a los cuales se les asigna un tema y deben de buscar los aspectos positivos o negativos. Luego se procede a realizar un debate con el docente como moderador.

Grupos de investigación. El grupo recibe un tema de investigación; se divide en subgrupos, para que proceden a investigar sobre la parte asignada, con el propósito de generar experticia en ese punto. Al final, intercambian la información entre todos, redactan un documento final.

Juegos de rol. El profesor asigna un tema determinado; especifica los roles de los estudiantes, cuyos roles son desempeñados durante los debates, las discusiones y las conclusiones.

Estudio de casos. El grupo recibe el resumen de un problema; los estudiantes logran los objetivos elaborados y propuestos por el profesor; el grupo realiza la exposición sobre sus resultados; se finaliza discutiendo y proponiendo soluciones.

Afiche. Se organizan subgrupos dentro de un grupo más grande; deben elaborar un afiche sobre un determinado tema, en cuyo afiche se registran los resultados de la discusión, luego son expuestos delante de todo el grupo.

\section{Entornos virtuales}

Un entorno virtual de aprendizaje es el escenario, donde un estudiante aprende, cuyos proceso es auto dirigido, donde se utiliza una serie de recursos que generan el aprendizaje activo, cooperativo, progresivo e independiente, en pro de la construcción de conocimientos y la adquisición de competencias personales y profesionales (Hiraldo, 2013). Desde la posición de Chan (2016), "la virtualización de la educación se presenta como un fenómeno en donde se actualizan los métodos educativos con el uso de TICs" (p.3).

Además Aguilar \& Otuyemi (2020) afirman: Los “entornos virtuales” también son definidos como recursos que complementan la gestión y la labor docente, dado que mejoran los procesos de enseñanza-aprendizaje y establecen la generación de actividades formativas orientadas a la adquisición de nuevos conocimientos y a la apropiación de los contenidos las herramientas virtuales pueden ser precisados como medios que complementan y apoyan el trabajo docente, ya que esta mejora y favorece el proceso de enseñanza - aprendizaje, además implanta actividades de formación con el objetivo de adquirir nuevos conocimientos (p.66). 
Martínez y Fernández (citados por Basantes et al., 2018) declaran que existen múltiples ventajas del uso del entorno virtual para la enseñanza; entre ellas: la manipulación y la exploración de fases de aprendizaje, la reflexión sobre el proceso de aprendizaje; la acomodación de los conocimientos nuevos a los ya adquiridos; la colaboración, discusión y cooperación con los estudiantes del aula y la extrapolación consciente de los conocimientos aprendidos a un contexto futuro de trabajo.

El uso de entornos virtuales en las aulas de clase favorece y apoya la participación de los docentes y los estudiantes, quienes intercambian información y participan en la resolución de problemas (Sierra et al., 2016).

Por otro lado, la expansión de internet y las TICs han transformado radicalmente la forma de aprender e interrelacionarse, en los diferentes ámbitos y en el contexto contemporáneo; proveen un sinnúmero de herramientas, recursos, medios y formatos, que permiten el uso de estrategias didácticas, con el propósito de facilitar la construcción de conocimientos; la posibilidad de utilizar la tecnología emergente, durante el proceso de enseñanza-aprendizaje, motivó que los entornos virtuales de aprendizaje replicaran el paradigma conductista, con sobreabundancia de información, propuestas de formación virtual sin ningún tipo de rigurosidad. En este sentido, se priorizó la tecnología sobre la pedagogía; la comunicación se relegó a segundo plano y la tasa de deserción de los estudiantes fue sumamente alta. Se aprecia que solo el 15\% de los estudiantes concluyen los cursos online, quedando debilitado el nivel de aceptación de la educación virtual (Basantes et al., 2018).

La incorporación de estrategias tecnológicas, en forma virtual en el campo educativo, ha provocado que el docente forme, renueve y genere nuevas destrezas, métodos, recursos y técnicas pedagógicas, cuyo propósito es aumentar el nivel de calidad de los estudiantes. Las estrategias didácticas, en el entorno virtual, conllevan a un proceso de asimilación y conocimiento pleno. Al implementarse las estrategias se diseñan actividades, para que el estudiante adquiera un aprendizaje, haciendo e interactuando, con métodos activos: exploración y procesamiento de información, proyectos, resolución de problemas, trabajo en equipo, foros de discusión; para que los estudiantes desplieguen capacidades: trabajo en equipo, autonomía y cooperación (Silva, 2017, citado por Palma et al., 2021). 


\section{Importancia de las estrategias y técnicas didácticas.}

\section{Para el estudiante}

\section{Potencia las habilidades}

Generalmente dentro de la presencialidad, incluso en la virtualidad, muchas veces, los estudiantes son partícipes de una educación pasiva; es decir, participan en el proceso en la condición de oyentes. Las técnicas didácticas intervienen, cambian el proceso de interacción, potencian las habilidades actitudinales, procedimentales y académicas, en el ámbito donde se desarrollan los estudiantes; también estas técnicas didácticas, en los alumnos, mejoran su confianza, el trabajo en equipo y la motivación, con el propósito de lograr el aprendizaje activo; por otro lado, estas estrategias potencian las habilidades para la adquisición del conocimiento (Mejia \& Lopez, 2017).

\section{Estimula el aprendizaje autónomo}

Los estudiantes, usando las técnicas y las estrategias didácticas, mejoran su aprendizaje autónomo, las necesidades, el acceso limitado, la interacción con bibliografías en la red, los debates virtuales que ayudan el aprendizaje autónomo.

\section{Facilita la interacción}

Durante mucho tiempo se ha requerido y buscado materializar la interacción voluntaria de los estudiantes, sin sentir presión de ningún tipo; este problema o deficiencia aún persiste; sin embargo, se han observado algunos cambios, por el uso y la aplicación de las estrategias y técnicas didácticas, las cuales promueven la interacción entre el docente-alumno, alumno-contenido y alumno-alumno (De Benito \& Salinas, 2005).

Promueve la creatividad

Al momento de ejecutar las técnicas didácticas muchos logran potenciar la creatividad ya que tienen que trabajar en equipo y además realizar trabajos propios. Es más en la educación de los niños este aspecto es más relevante ya que los pequeños están más activos en la formación de su creatividad, es así que en la experimentación realizada por (Rivas, 2016) demuestra que los niños desarrollaron creatividad.

\section{Para el docente}

Mejora la relación con sus estudiantes

Con el desarrollo de la interacción, gracias a las estrategias y técnicas didácticas, los docentes son favorecidos mediante la confianza y la mejor relación entre él y el 
estudiante, aportando mayor estímulo para el aprendizaje; además, estas técnicas permiten que el docente identifique problemáticas en el aprendizaje del estudiante.

Garantiza la calidad educativa

El docente, usando las estrategias y las técnicas didácticas, garantiza la calidad educativa, porque, dentro de la virtualidad, los materiales por sí mismos no pueden enseñar; es decir, los materiales brindan información; sin embargo, los estudiantes no tienen la certeza de identificación de lo correcto; para advertir lo correcto, el docente entra en acción, en constante seguimiento al estudiante; también elige la estrategia didáctica correcta, con el propósito de lograr una educación de calidad (Camacho et al., 2017).

\section{Promueve motivación}

Un docente, mediante el uso correcto de la estrategia y técnica didáctica necesaria y pertinente, logra la motivación imprescindible para sus estudiantes. Es muy importante que el docente use las estrategias y las técnica didácticas, con el propósito de lograr los objetivos pedagógicos, en un contexto de motivación del estudiante (Romero, 2009).

\section{Conclusiones}

Por lo expuesto anteriormente, se determina que el uso de estrategias y técnicas didácticas, en un entorno virtual, es imprescindible para los protagonistas del proceso enseñanza aprendizaje: docentes y estudiantes, porque permite que los docentes puedan mejorar sus estilos de enseñanza, motivando en los estudiantes el desarrollo de un pensamiento creativo y crítico. Asimismo, ofrecen muchas ventajas; por ejemplo, la potenciación de habilidades y el aprendizaje autónomo, la manipulación y exploración de fases de aprendizaje, la discusión y cooperación inmediata con los estudiantes. El uso de estrategias y técnicas didácticas, adecuadas y pertinentes, permitirán una vinculación mayor entre el docente y el alumno, y, por ende, un aprendizaje significativo en esta era virtual.

No es una novedad saber que se necesitan nuevos modelos didácticos para la enseñanza virtual; es importante, necesaria y vital la aplicación de estrategias y técnicas didácticas. Este ámbito implica y exige una mente dispuesta a conducirse pedagógicamente, construyendo verdaderamente una revolución del sistema educativo en esta era virtual, cuyos docentes sean cada vez más actualizados con la información y el manejo de las TIC, transmitiendo, guiando y reconstruyendo el conocimiento en los estudiantes. 


\section{Referencias}

Aguilar, L., \& Otuyemi, E. (2020). Análisis documental: importancia de los entornos virtuales en los procesos educativos en el nivel superior. Tecnología, Ciencia y Educación, 17, 57-77. https://doi.org/https://doi.org/10.51302/tce.2020.485

Basantes, A., Naranjo, M., \& Ojeda, V. (2018). Metodología PACIE en la Educación Virtual: una experiencia en la Universidad Técnica del Norte Andrea. Formacion Universitaria, 11(2), 35-44. https://doi.org/10.4067/S0718-50062018000200035

Camacho, M., Lara, Y., \& Sandoval, G. (2017). Estrategias de aprendizajes para Entornos Virtuales María.

https://acceso.virtualeduca.red/documentos/ponencias/puerto-rico/1399-63cb.pdf

Chan, M. (2016). La virtualización de la educación superior en América Latina: entre tendencias y paradigmas. Revista de Educación a Distancia (RED), 48, 1-32. https://doi.org/10.6018/red/48/1

De Benito, B., \& Salinas, J. (2005). Situaciones didácticas en los entornos virutales de enseñanza-aprendizaje (EVEA) en la enseñanza superior: elaboración de un instrumento de análisis. In EDUTEC05 (pp. 1-18).

https://www.researchgate.net/publication/232242321_Situaciones_didacticas_en_1 os_entornos_virutales_de_ensenanzaaprendizaje_EVEA_en_la_ensenanza_superior_elaboracion_de_un_instrumento_d e analisis

Delgado, M., \& Solano, A. (2009). Estrategias didácticas creativas en entornos virtuales para el aprendizaje. Revista INIE, 9, 1-21.

http://euaem1.uaem.mx/bitstream/handle/123456789/1538/estrategias.pdf?sequenc $\underline{\mathrm{e}=1 \& \text { is }}$

Feo, R. (2010). Orientaciones Básicas para el diseño de Estrategias Didácticas. Instituto Pedagógico de Miranda José Manuel Siso Martínez, 220-236. https://doi.org/10.1016/S0140-6736(87)90255-8

Hiraldo, R. (2013). Uso de los entornos virtuales de aprendizaje en la educación a distancia. EDUTEC Costa Rica, 1-14. https://www.uned.ac.cr/academica/edutec/memoria/ponencias/hiraldo_162.pdf 
Hoffman, A. F., Ledesma, R., \& Liporace, M. F. (2017). Estilos y estrategias de aprendizaje en estudiantes universitarios de Buenos Aires. Revista de Psicologia (Peru), 35(2), 535-573. https://doi.org/10.18800/psico.201702.006

Labrador, J., \& Andreu, A. (2008). Metodologias Activas. Editorial UPV. http://www.upv.es/diaal/publicaciones/Andreu-Labrador12008_Libro Metodologias_Activas.pdf

Maria, T. (2020). Estrategias de Aprendizaje. RIESCE, 1-2. http://ri.utn.edu.mx//handle/123456789/225

Marsiglia, R., Llamas, J., \& Fuentes, E. (2020). Las estrategias de enseñanza y los estilos de aprendizaje una aproximación al caso de la licenciatura en educación de la Universidad de Cartagena (Colombia). Formacion Universitaria, 13(1), 27-34. https://doi.org/10.4067/S0718-50062020000100027

Mejia, L., \& Lopez, D. (2017). Una mirada a las estrategias y técnicas didácticas en la educación en ingeniería. Entre Ciencia e Ingeniería, 11(21), 123-132. http://www.scielo.org.co/scielo.php?script=sci_arttext\&pid=S1909$\underline{83672017000100123 \& \operatorname{lng}=\mathrm{es} \& \mathrm{nrm}=\mathrm{iso}}$

Palma, E., Renteria, F., \& Castro, J. (2021). Estrategia didáctica en el entorno virtual para la enseñanza de electrónica. Revista Clake Education, 2. http://revistaclakeeducation.com/ojs/index.php/Multidisciplinaria/article/view/65

Pamplona, J., Cuesta, J., \& Cano, V. (2019). Estrategias De Enseñanza Del Docente En Las Áreas Básicas: Una Mirada Al Aprendizaje Escolar. Revista Eeuthera, 21, 13 33. https://doi.org/10.17151/eleu.2019.21.2

Rivas, Y. (2016). Técnicas didácticas para el proceso de socialización en Educación Inicial.Educ@ción En Contexto, 3, 119-135. https://dialnet.unirioja.es/descarga/articulo/6296703.pdf

Romero, G. (2009). La utilización de estrategias didácticas en clase. Innovación y Experiencias Educativas, 1-8.

https://archivos.csif.es/archivos/andalucia/ensenanza/revistas/csicsif/revista/pdf/Nu mero_23/GUSTAVO_ADOLFO_ROMERO_BAREA02.pdf

Rosales, J. (n.d.). Estrategias didácticas. Universidad Nacional Autónoma de México. 
http://www.formaciondocente.com.mx/PublicacionesPedagogicas/Articulos/Estrate gias Didacticas.pdf

Sierra, J., Bueno, I., \& Monroy, S. (2016). Análisis del uso de las tecnologías TIC por parte de los docentes de las Instituciones educativas de la ciudad de Riohacha. Revista Omnia, 22(2), 50-64.

https://www.redalyc.org/articulo.oa?id=73749821005

Silva, J., \& Maturana, D. (2017). Una propuesta de modelo para introducir metodologías activas en educación superior. Innovación Educativa, 17(73), 117131. http://www.scielo.org.mx/scielo.php?script=sci_arttext\&pid=S166526732017000100117\&lng=es\&tlng=es. 